\title{
Photovoltaic effect generated by spin-orbit interactions
}

\author{
O. Entin-Wohlman,,${ }^{1, *}$ R. I. Shekhter,${ }^{2}$ M. Jonson, ${ }^{2}$ and A. Aharony ${ }^{1}$ \\ ${ }^{1}$ Raymond and Beverly Sackler School of Physics and Astronomy, Tel Aviv University, Tel Aviv 69978, Israel \\ ${ }^{2}$ Department of Physics, University of Gothenburg, SE-412 96 Göteborg, Sweden
}

(Dated: April 1, 2020)

\begin{abstract}
An AC electric field applied to a junction comprising two spin-orbit coupled weak links connecting a quantum dot to two electronic terminals is proposed to induce a DC current and to generate a voltage drop over the junction if it is a part of an open circuit. This photovoltaic effect requires a junction in which mirror reflection-symmetry is broken. Its origin lies in the different fashion inelastic processes modify the reflection of electrons from the junction back into the two terminals, which leads to uncompensated DC transport. The effect can be detected by measuring the voltage drop that is built up due to that DC current. This voltage is an even function of the frequency of the AC electric field.
\end{abstract}

1. Introduction. Electric weak links made of materials with strong spin-orbit interactions open a promising way to achieve spin-dependent transport of electrons. In the particular case of the Rashba spin-orbit coupling [1], the interaction can be tuned electrostatically [2-4] or mechanically $[5,6]$. This coupling obeys time-reversal symmetry which prevents spin splitting of electron transport in two-terminal junctions [7], in most cases eliminating the possibility to manipulate electronic conduction through Rashba weak links. Spin-orbit interactions do, however, have an effect on spin-polarized electrons in magnetic materials [8-10], and on electrons subjected to external magnetic fields [11-16]. Here we propose that imposing a time dependence on the effective magnetic fields induced by the spin-orbit coupling offers another means to destroy time-reversal symmetry of twoterminal junctions. In particular we predict that timedependent Rashba interactions generate a DC electric current through unbiased junctions.

Coherent electronic transport in response to periodic modulations of the shape of quantum dots or of other potential parameters of mesoscopic junctions has been attracting considerable interest $[17,18]$ following the seminal paper by Thouless [19], who showed that a slow periodic variation of the potential landscape may yield quantized and non-dissipative particle transport in unbiased junctions-a phenomenon termed "adiabatic quantum pumping". Adiabatic pumping of spin currents resulting from periodic modulations of the shape of a spinorbit coupled junction has been discussed as well [20], also as a result of temporal modulations of the Rashba interaction [21-24]. However, the possibility to induce a DC particle current by such modulations in the absence of a bias voltage was not considered.

DC charge transport driven by time-dependent spinorbit coupling is an alternative to the pumping of charge caused by tuning periodically the potential landscape of mesoscopic structures. It occurs in inhomogeneous junctions in which mirror reflection-symmetry is violated. In an unbiased junction no net current flows when the spinorbit interaction is static, even in an asymmetric device:

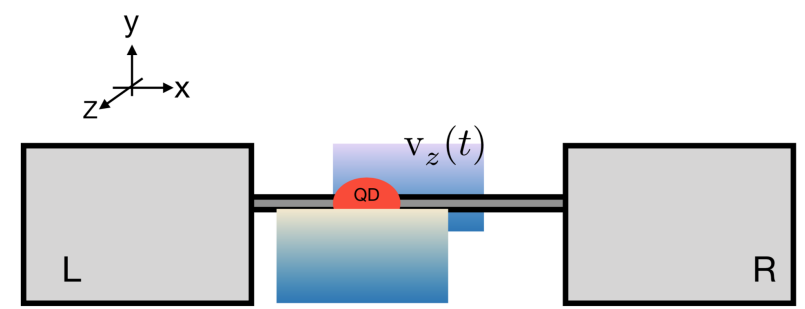

FIG. 1: (Color online.) Illustration of the model system. A quantum dot, represented by a localized energy level, is attached by two weak links lying in the $x-y$ plane to two reservoirs, denoted $L$ and $R$. An AC electric field along $\hat{\mathbf{z}}$, whose amplitude oscillates with frequency $\Omega$, induces a Rashba spinorbit interaction in the links.

transport of electrons incident from the two opposite reservoirs is fully equilibrated. In fact, a static spinorbit coupling, which results in a unitary evolution of the spinor wave-function, does not modify the DC transport. However, unitarity is destroyed by the time dependence that entails additional reflection processes due to inelastic tunneling. These in general differ for the two opposite directions in which electrons can be reflected from the junction, leading to uncompensated electronic transport. To elaborate on this general statement we refer to the device illustrated in Fig. 1: a quantum dot represented by a single level of energy $\epsilon$ is connected by spin-orbit coupled weak links to left and right reservoirs. Due to the Aharonov-Casher effect [25], the tunneling matrix elements attain unitary-matrix (in spin space) phase factors [26], denoted below by $V_{L(R)}$ for tunneling through the left (right) link. When these are time dependent, the reflection, say to the left direction, is then modified by factors of the form

$$
\int^{t} d t^{\prime}\left[V_{L}^{\dagger}(t) e^{i(\epsilon-\omega+i \Gamma)\left(t-t^{\prime}\right)} V_{L}\left(t^{\prime}\right)+\text { c.c. }\right],
$$

where $\Gamma$ is the width of the resonance formed on the dot (using $\hbar=1$ ). This form pertains to tunneling from the left lead to the dot, accomplished at time $t^{\prime}$, followed 
by a time evolution of the electronic wave-function on the dot during the time interval $t-t^{\prime}$, and then tunneling back to the left lead at time $t$. One observes that in the static case, where $V_{L}^{\dagger} V_{L}=1$, the integral (1) yields the usual Breit-Wigner density of states on the dot, $2 \Gamma /\left[(\omega-\epsilon)^{2}+\Gamma^{2}\right]$. For a Rashba interaction that varies periodically with frequency $\Omega$, the reflection comprises multiple inelastic channels with emission and absorption of $n \Omega$ energy quanta, which shift the resonance above and below $\epsilon$. This complex modification of the reflection may differ for the opposite directions of the junction, leading to a net DC current. Below we show that such a difference can indeed result from the Rashba interaction when the lengths of the two weak links are not identical.

2. Details of the model. The Rashba interaction in the links is induced by external electric fields, which can be polarized in various ways. Here we focus on the simplest one of a longitudinal field (along the $\hat{\mathbf{z}}$ direction), whose amplitude oscillates with frequency $\Omega$ (see Fig. 1). The Aharonov-Casher phase factor multiplying the tunneling amplitude through a link of length $d$ along $\hat{\mathbf{d}}$ is then [27, 28]

$$
\exp \left[i \varphi_{A C}(t)\right]=\exp \left[i k_{\mathrm{so}} d \cos (\Omega t) \hat{\mathbf{z}} \times \hat{\mathbf{d}} \cdot \boldsymbol{\sigma}\right],
$$

where $\boldsymbol{\sigma}=\left(\sigma_{x}, \sigma_{y}, \sigma_{z}\right)$ is the vector of the Pauli matrices, and $k_{\mathrm{so}}$ is the Rashba coupling. For the geometry of Fig. 1 the Aharonov-Casher phase factors are

$$
\begin{aligned}
V_{L(R)}(t) & =\cos \left[k_{\mathrm{so}} d_{L(R)} \cos (\Omega t)\right] \\
& +i \sin \left[k_{\mathrm{so}} d_{L(R)} \cos (\Omega t)\right] \boldsymbol{\sigma} \cdot \hat{\mathbf{e}}_{L(R)},
\end{aligned}
$$

where $d_{L(R)}$ is the length of the link connecting the dot to the left (right) reservoir. For links along the $\hat{\mathbf{x}}$-direction (Fig. 1), the effective magnetic fields created by the Rashba interaction are along $\hat{\mathbf{e}}_{L}=-\hat{\mathbf{y}}$ and $\hat{\mathbf{e}}_{R}=\hat{\mathbf{y}}$.

The entire junction is described by the Hamiltonian

$$
\mathcal{H}(t)=\mathcal{H}_{0}+\mathcal{H}_{\text {tun }}(t),
$$

where the time-independent $\mathcal{H}_{0}$ pertains to the decoupled system, i.e., two separate reservoirs and a quantum dot,

$$
\mathcal{H}_{0}=\sum_{\sigma} \epsilon d_{\sigma}^{\dagger} d_{\sigma}+\sum_{\mathbf{k}, \sigma} \epsilon_{k} c_{\mathbf{k} \sigma}^{\dagger} c_{\mathbf{k} \sigma}+\sum_{\mathbf{p}, \sigma} \epsilon_{p} c_{\mathbf{p} \sigma}^{\dagger} c_{\mathbf{p} \sigma} .
$$

The first term in Eq. (5) describes the decoupled dot, with $d_{\sigma}^{\dagger}\left(d_{\sigma}\right)$ being the creation (annihilation) operator of an electron of energy $\epsilon$ in the spin state $|\sigma\rangle$. The other two terms describe the decoupled electronic reservoirs, assumed to comprise non-polarized free electrons. There, $c_{\mathbf{k} \sigma}^{\dagger}\left(c_{\mathbf{k} \sigma}\right)$ creates (annihilates) a particle with energy $\epsilon_{k}$ $\left(\epsilon_{p}\right)$, momentum $\mathbf{k}(\mathbf{p})$, and spin $\sigma$ in the left (right) lead. The tunneling Hamiltonian reads

$$
\begin{aligned}
& \mathcal{H}_{\text {tun }}(t)=\sum_{\alpha=L, R} \mathcal{H}_{\text {tun }}^{\alpha}(t) \\
& =\sum_{\alpha} J_{L(R)} \sum_{\sigma, \sigma^{\prime}}\left\{\left[V_{L(R)}(t)\right]_{\sigma \sigma^{\prime}} \sum_{\mathbf{k}(\mathbf{p})} c_{\mathbf{k}(\mathbf{p}) \sigma^{\dagger}}^{\dagger} d_{\sigma^{\prime}}+\text { H.c. }\right\}
\end{aligned}
$$

The tunneling amplitudes, characterized by the energy $J_{L(R)}$, are assumed to be given by their values at the Fermi energy.

3. Currents in the time domain. Within the Keldysh technique $[29,30]$ the particle current, say into the left lead, is conveniently expressed in terms of the Green's function on the $\operatorname{dot}[31] G_{d d}\left(t, t^{\prime}\right)$ (a matrix in spin space),

$$
\begin{gathered}
I_{L}(t) \equiv \frac{d}{d t} \sum_{\mathbf{k}} \sum_{\sigma}\left\langle c_{\mathbf{k} \sigma}^{\dagger} c_{\mathbf{k} \sigma}\right\rangle=\int d t_{1} \operatorname{Tr}\left\{\Sigma_{L}\left(t, t_{1}\right) G_{d d}\left(t_{1}, t\right)\right. \\
\left.-G_{d d}\left(t, t_{1}\right) \Sigma_{L}\left(t_{1}, t\right)\right\}^{<},
\end{gathered}
$$

where the angular brackets denote quantum averaging. The superscript $<$ indicates the lesser Green's function, and $\Sigma_{L}\left(t, t^{\prime}\right)$ is the self energy due to the coupling of the dot to the left reservoir,

$$
\Sigma_{L}\left(t, t^{\prime}\right)=J_{L}^{2} V_{L}^{\dagger}(t) g_{L}\left(t, t^{\prime}\right) V_{L}\left(t^{\prime}\right),
$$

where $g_{L}\left(t, t^{\prime}\right)$ is the Green's function of the decoupled left reservoir. Green's functions without a superscript represent all three Keldysh Green's functions, the lesser, and the retarded and advanced ones (marked by the superscripts $r$ and $a$ ). The expression in Eq. (7) can be worked out explicitly in the wide-band limit [32, 33], where the densities of states in each of the reservoirs are approximated by their value on the Fermi surface. The self energy $\Sigma_{L}^{r(a)}\left(t, t^{\prime}\right)$ is then proportional to the unit matrix in spin space, with

$$
\Sigma_{L}^{r(a)}\left(t, t^{\prime}\right)=\mp i \Gamma_{L} \delta\left(t-t^{\prime}\right)
$$

where

$$
\Gamma_{L}=\pi \mathcal{N}_{L} J_{L}^{2}
$$

is the (partial) width of the resonance formed on the dot due to the coupling with the left reservoir and $\mathcal{N}_{L}$ denotes the density of states of the left lead on the Fermi surface. The total width of the resonance on the dot is $\Gamma=\Gamma_{L}+\Gamma_{R}$. The lesser self energy is a matrix in spin space,

$$
\Sigma_{L}^{<}\left(t, t^{\prime}\right)=2 i \Gamma_{L} \int \frac{d \omega}{2 \pi} e^{-i \omega\left(t-t^{\prime}\right)} f(\omega) V_{L}^{\dagger}(t) V_{L}\left(t^{\prime}\right) .
$$

Here $f(\omega)=\{\exp [\beta(\omega-\mu)]+1\}^{-1}$ is the equilibrium Fermi distribution, with the inverse temperature $\beta$ and the chemical potential $\mu$ being identical for the two reservoirs. $\left[\Sigma_{R}\left(t, t^{\prime}\right)\right.$ is obtained from these expressions by changing $L$ to $R$.]

The explicit expression for $I_{L}(t)$ is found by applying the Langreth rules [29] to Eq. (7),

$$
\begin{aligned}
& I_{L}(t)=2 \Gamma_{L} \operatorname{Tr}\left\{-i G_{d d}^{<}(t, t)-\int \frac{d \omega}{2 \pi} f(\omega)\right. \\
& \left.\times \int^{t} d t_{1}\left[e^{-i(\epsilon-\omega+i \Gamma)\left(t_{1}-t\right)} V_{L}^{\dagger}(t) V_{L}\left(t_{1}\right)+\text { c.c. }\right]\right\} .
\end{aligned}
$$


The equal-time lesser Green's function $-i G_{d d}^{<}(t, t)$, which yields the occupation of the dot, is

$$
-i G_{d d}^{<}(t, t)=\int \frac{d \omega}{\pi} f(\omega)\left[\Gamma_{L} \mathrm{w}_{L}(\omega, t)+\Gamma_{R} \mathrm{w}_{R}(\omega, t)\right],
$$

where

$$
\begin{aligned}
& \mathrm{w}_{L}(\omega, t)=\int^{t} d t_{1} \int^{t} d t_{2} e^{-i \omega\left(t_{1}-t_{2}\right)} \\
& \times e^{-i(\epsilon-i \Gamma)\left(t-t_{1}\right)} e^{-i(\epsilon+i \Gamma)\left(t_{2}-t\right)} V_{L}^{\dagger}\left(t_{1}\right) V_{L}\left(t_{2}\right),
\end{aligned}
$$

with an analogous expression for $w_{R}(\omega, t)$ (more details are given in Ref. 33). Thus, integrals of the form (1) determine the explicit expressions for the current.

Using the expansion [34]

$$
e^{i \zeta \cos (\phi)}=\sum_{n=-\infty}^{\infty} i^{n} J_{n}(\zeta) e^{i n \phi},
$$

where $J_{n}(\zeta)$ in the Bessel function of integer order $n$, one finds (see Ref. 33 for details)

$$
\begin{aligned}
\mathrm{w}_{L}(\omega, t) & =\mid J_{0}\left(k_{\mathrm{so}} d_{L}\right) D(\omega) \\
& +\left.\sum_{n=1}^{\infty}(-1)^{n} J_{2 n}\left(k_{\mathrm{so}} d_{L}\right) F_{2 n}(\omega, t)\right|^{2} \\
& +\left|\sum_{n=0}^{\infty}(-1)^{n} J_{2 n+1}\left(k_{\mathrm{so}} d_{L}\right) F_{2 n+1}(\omega, t)\right|^{2},
\end{aligned}
$$

where

$$
D(\omega)=i /[\omega-\epsilon+i \Gamma]
$$

is the Breit-Wigner resonance on the dot, and

$$
F_{n}(\omega, t)=D(\omega-n \Omega) e^{i n \Omega t}+D(\omega+n \Omega) e^{-i n \Omega t}
$$

is an even function of $\Omega$ that contains the contributions of the inelastic processes. The second term on the righthand side of Eq. (12) is found in a similar fashion [33]. The particle current is then

$$
\begin{aligned}
I_{L}(t)= & 4 \Gamma_{L} \Gamma_{R} \int \frac{d \omega}{\pi} f(\omega)\left[\mathrm{w}_{R}(\omega, t)-\mathrm{w}_{L}(\omega, t)\right] \\
& -2 \Gamma_{L} \int \frac{d \omega}{\pi} f(\omega) \frac{d \mathrm{w}_{L}(\omega, t)}{d t} .
\end{aligned}
$$

One notes that $I_{L}(t)+I_{R}(t)$ [the latter is obtained by interchanging $L$ with $R$ in Eq. (19)] equals the time derivative of $-\operatorname{Tr}\left\{i G_{d d}^{<}(t, t)\right\}$ [Eq. (13)] which is the occupation on the dot; i.e., charge is conserved in the junction. Note also that for $\Omega=0 \mathrm{Eq}$. (16) becomes $\mathrm{w}_{L}(\omega)=\left[\cos ^{2}\left(k_{\mathrm{so}} d_{L}\right)+\sin ^{2}\left(k_{\mathrm{so}} d_{L}\right)\right]|D(\omega)|^{2}=|D(\omega)|^{2}$ which depends neither on the length $d_{L}$ nor on the spinorbit coupling $k_{\mathrm{so}}$. In that case the first term on the right-hand side of Eq. (19) vanishes, and there is no DC particle flow in an unbiased junction.

4. DC electromotive force generated by time-dependent Rashba interaction. The current $I_{L}(t)$ comprises a static term, in addition to the time-dependent one. Obviously the derivative $d \mathrm{w}_{L}(t) / d t$ depends on time; but $\mathrm{w}_{L}$ (and similarly $\mathrm{w}_{R}$ ) contains a static term, $\mathrm{w}_{L, \mathrm{~s}}$, which takes a particularly simple form,

$$
\mathrm{w}_{L, \mathrm{~s}}(\omega)=\sum_{n=-\infty}^{\infty} J_{n}^{2}\left(k_{\mathrm{so}} d_{L}\right)|D(\omega-n \Omega)|^{2}
$$

which is even in $\Omega$. As a result, the DC particle current through the junction is

$$
\begin{aligned}
I_{D C} & =\int \frac{d \omega}{\pi} 4 \Gamma_{L} \Gamma_{R}|D(\omega)|^{2} \\
& \times \sum_{n=-\infty}^{\infty} f(\omega+n \Omega)\left[J_{n}^{2}\left(k_{\mathrm{so}} d_{R}\right)-J_{n}^{2}\left(k_{\mathrm{so}} d_{L}\right)\right] .
\end{aligned}
$$

(The time-dependent parts of the currents can be found in Ref. 33.) Figure 2 portrays the current vs. $k_{\mathrm{so}} d_{R}$ at a fixed value of $k_{\mathrm{so}} d_{L}$, as calculated from Eq. (21) for several values of temperature and $\Omega$. The oscillations, at low temperatures, reflect the oscillatory length-dependence of the effect of the Rashba interaction [27]. These oscillations disappear gradually as the temperature is raised. At low temperatures and large $\Omega$ 's, $I_{D C}$ is dominated by the oscillations of the zeroth and first order Bessel functions.

The appearance of DC electronic charge transport in the absence of a bias voltage across the device is a manifestation of an electromotive force acting in the electric circuit. In our case, the force relies on the electronic spin, and drives the electrons' flow through a junction subject to a time-dependence spin-orbit interaction. The driving occurs due to the fermionic nature of the electrons which imposes constraints on the inelastic spin-scattering induced by the time-dependent Rashba interaction: some of the inelastic scattering channels become partly blocked due to the Pauli principle. This is why the unitarity of spin transmission, which would hold if all inelastic transmission channels would be equally open, is broken [35]. The peculiar photovoltaic effect discussed above manifests itself in inhomogeneous devices with a well-defined direction of the inhomogeneity along the direction of the current flow. The single-dot tunneling device studied here, in which the reflection asymmetry is generated by the different lengths of the links (in conjunction with the Rashba coupling), is an example of such an inhomogeneity.

One may propose a simple procedure to measure the spin-orbit-induced electromotive force. Suppose that the spin-orbit coupled weak link (which contains the dot) is an element of an open electric circuit. Then the spindriven electromotive force would lead to an accumulation 

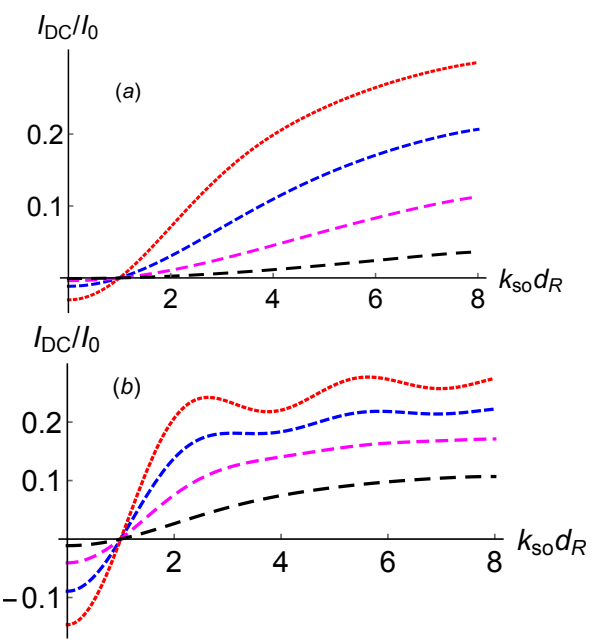

FIG. 2: The particle current $I_{\mathrm{DC}}$, normalized to $I_{0}=$ $\left(4 \Gamma_{L} \Gamma_{R}\right) /(\Gamma \pi \hbar)$, calculated from Eq. (21) as a function of $k_{\mathrm{so}} d_{R}$ for $k_{\mathrm{so}} d_{L}=1.0, \epsilon-\mu=0.5 \Gamma$; (a) $\Omega=0.5 \Gamma$, (b) $\Omega=2.0 \Gamma$. The increasing dash lengths correspond to $\beta \Gamma=10.0,2.0,1.0$, and 0.5 .

of extra charges of opposite signs on the two terminals, and in turn to the building up of a voltage drop across the junction. A steady-state configuration would then emerge, provided that the ohmic current generated by this voltage drop compensates the DC current due to the electromotive force induced by the Rashba interaction.

Thus, a novel photovoltaic effect can be predicted: a microwave field applied to a gate electrode (see Fig. 1) induces a voltage drop across the junction. A simple analytical estimate of the voltage signal can easily be obtained for a weak Rashba coupling, $k_{\text {so }} d_{L(R)} \ll 1$ : In the absence of the spin-orbit interaction, a bias voltage $V$ on the junction gives rise to a DC particle current [30]

$$
I_{\mathrm{DC}}=\frac{4 \Gamma_{L} \Gamma_{R}}{\pi \hbar} \frac{e V}{\epsilon_{0}^{2}+\Gamma^{2}} \sim \frac{4 \Gamma_{L} \Gamma_{R}}{\pi \epsilon_{0}^{2}} \frac{e V}{\hbar},
$$

where $\epsilon_{0} \equiv \epsilon-\mu$ is assumed to be much larger than $\Gamma$ in the last step ( $\hbar$ was re-introduced into the expressions for the following estimates). On the other hand, according to Eq. (21) at small spin-orbit coupling, the oscillating Rashba interaction generates the zero-temperature particle DC current

$$
\begin{aligned}
& \left.I_{\mathrm{DC}}\right|_{T=0}=\frac{4 \Gamma_{L} \Gamma_{R}}{\pi \hbar \Gamma} \frac{k_{\mathrm{So}}^{2}\left[d_{L}^{2}-d_{R}^{2}\right]}{2} \\
& \times\left[\frac{1}{2}\left(\arctan \frac{\epsilon_{0}+\Omega}{\Gamma}+\arctan \frac{\epsilon_{0}-\Omega}{\Gamma}\right)-\arctan \frac{\epsilon_{0}}{\Gamma}\right],
\end{aligned}
$$

which becomes, for $\epsilon_{0} \gg \Gamma$ and $\Omega<\epsilon_{0}$

$$
\left.I_{\mathrm{DC}}\right|_{T=0} \sim \frac{4 \Gamma_{L} \Gamma_{R}}{\pi \epsilon_{0}^{2}} k_{\mathrm{so}}^{2}\left[d_{R}^{2}-d_{L}^{2}\right] \frac{(\hbar \Omega)^{2}}{2 \hbar \epsilon_{0}} .
$$

Thus, the voltage drop $V_{\text {em }}$ generated by the electromotive force is

$$
V_{\mathrm{em}}=k_{\mathrm{so}}^{2}\left(d_{R}^{2}-d_{L}^{2}\right)(\hbar \Omega)^{2} /\left(2 e \epsilon_{0}\right) .
$$

Similar considerations for the oscillatory lengthdependence pertain to the case where the Rashba interaction is induced by mechanical vibrations of the nanowire forming the link $[5,6]$.

Our model system could be implemented by, e.g., three in-line, side-gated InAs nanowires [36]. The left and right nanowires of length $d_{L}$ and $d_{R}$ would serve as weak links and be in tunneling contact with, respectively, the sourceand drain electrodes as well as with the short, central nanowire, which would serve as a quantum dot. One of the two gates on either side of the weak links would be excited by a microwave field that creates an AC gate voltage, $V_{a c} \cos (\Omega t)$ [37], while a static voltage on the the two gates on either side of the quantum dot would be used to tune the energy levels in the dot.

With a distance of $\sim 200 \mathrm{~nm}$ between the side gates [36] a microwave-generated amplitude of $V_{a c}=1 \mathrm{~V}$ on the side gates would produce a transverse electric field amplitude of $\sim 50 \mathrm{kV} / \mathrm{cm}$ in the wires, corresponding to a Rashba parameter $\alpha_{R}=\hbar^{2} k_{\mathrm{so}} / m^{*} \sim 50 \mathrm{meV} \cdot \AA$ [38] and, using $m^{*}=0.023 m_{e}$, a Rashba coupling $k_{\text {so }} \sim 2$. $10^{-3}(\mathrm{~nm})^{-1}$ in the weak links. Assuming $d_{L} \sim d_{R} \sim$ $250 \mathrm{~nm}$ and microwave frequency of $2 \pi \times 100 \mathrm{GHz}$ (so that $\hbar \Omega$ is of the order of the energy level $\epsilon_{0} \sim 1 \mathrm{meV}$ [with respect to the chemical potential]) one finds $V_{\mathrm{em}} \sim$ $5 \mu \mathrm{V}$; thus the photovoltaic voltage in response to the microwave field seems to be measurable. Using the same parameter estimates the particle current, Eq. (24), is $\sim 5 \cdot 10^{7} \mathrm{~s}^{-1}$, corresponding to a charge current $\sim 10 \mathrm{pA}$. 5. Summary. We have found that the spin-orbit (Rashba) interaction confined to an electric weak link, which - when static - has no significant effect on DC transport of two-terminal devices, may act as a source of DC currents when generated by a periodic electric field. This electric field renders the Rashba interaction time dependent, breaking the unitarity of the spin transmission by generating inelastic transmission channels. We have shown that this loss of unitarity appears as additional contributions to the backscattering [see Eqs. (1) and (14)]. An estimate of the generated voltage drop in an open circuit suggests that it can be detected experimentally.

The effect we find is due to modifications of the probabilities for electron reflections, which are different for electrons approaching the junction from opposite directions; nonetheless, it is not related to quantum pumping [19]. The origin of the latter are different time-dependent phases of the instantaneous reflection amplitudes [18], whereas a straightforward calculation of the instantaneous scattering matrix for the junction illustrated in Fig. 1 shows that the reflection amplitudes do not depend on time. This is because $V_{L}^{\dagger}(t) V_{L}(t)=1$ due to the unitarity of the Aharonov-Casher phase factor. In our case, the reflections are modified by Aharonov-Casher phase factors at different times, and necessitate the inclusion of the inelastic dynamics on the dot. 
This research was partially supported by the Israel Science Foundation (ISF), by the infrastructure program of Israel Ministry of Science and Technology under contract 3-11173, and by the Pazy Foundation. We acknowledge the hospitality of the PCS at IBS, Daejeon, Korea, and Zhejiang University, Hangzhou, China, where part of this work was supported by IBS funding number (IBS-R024D1).

* Electronic address: entin@tau.ac.il

[1] E. I. Rashba, Properties of semiconductors with an extremum loop .1. Cyclotron and combinational resonance in a magnetic field perpendicular to the plane of the loop, Fiz. Tverd. Tela (Leningrad) 2, 1224 (1960) [Sov. Phys. Solid State 2, 1109 (1960)]; Y. A. Bychkov and E. I. Rashba, Oscillatory effects and the magnetic susceptibility of carriers in inversion layers, J. Phys. C 17, 6039 (1984).

[2] J. Nitta, T. Akazaki, H. Takayanagi, and T. Enoki, Gate Control of Spin-Orbit Interaction in an Inverted In $_{0.53} G_{0.47} A s / \operatorname{In}_{0.52} A l_{0.48}$ As Heterostructure, Phys. Rev. Lett. 78, 1335 (1997).

[3] Y. Sato, T. Kita, S. Gozu, and S. Yamada, Large spontaneous spin splitting in gate-controlled two-dimensional electron gases at normal $\operatorname{In}_{0.75} \mathrm{Ga}_{0.25} \mathrm{As} / \operatorname{In}_{0.75} A l_{0.25} \mathrm{As}$ heterojunctions, J. Appl. Phys. 89, 8017 (2001).

[4] A. J. A. Beukman, F. K. de Vries, J. van Veen, R. Skolasinski, M. Wimmer, F. Qu, D. T. de Vries, B. M. Nguyen, W. Yi, A. A. Kiselev, M. Sokolich, M. J. Manfra, F. Nichele, C. M. Marcus, and L. P. Kouwenhoven, Spin-orbit interaction in a dual gated InAs/GaSb quantum well, Phys. Rev. B 96, 241401(R) (2017).

[5] R. I. Shekhter, O. Entin-Wohlman, and A. Aharony, Suspended nanowires as mechanically controlled Rashba spin splitters, Phys. Rev. Lett. 111, 176602 (2013).

[6] M. Jonson, R. I. Shekhter, O. Entin-Wohlman, A. Aharony, H. C. Park, and D. Radić, Mechanically driven spin-orbit-active weak links, Low Temp. Phys. 44, 1228 (2018) [Fiz. Niz. Temp. 44, 1577 (2018)].

[7] J. H. Bardarson, A proof of the Kramers degeneracy of transmission eigenvalues from antisymmetry of the scattering matrix, J. Phys. A: Math. Theor. 41, 405203 (2008).

[8] S. Datta and B. Das, Electronic analog of the electro-optic modulator, Appl. Phys. Lett. 56, 665 (1990).

[9] A. Aharony, O. Entin-Wohlman, K. Sarkar, R. I. Shekhter, and M. Jonson, Effects of different lead magnetizations on the Datta-Das spin field-effect transistor, J. Phys. Chem. C 123, 11094 (2019) and references therein.

[10] R. I. Shekhter, O. Entin-Wohlman, and A. Aharony, Mechanically controlled spin-selective transport, Phys. Rev. B 90045401 (2014).

[11] A. G. Aronov and Y. B. Lyanda-Geller, Spin-Orbit Berry Phase in Conducting Rings, Phys. Rev. Lett. 70, 343 (1993).

[12] J. Splettstoesser, M. Governale, and J. König, Adiabatic charge and spin pumping through quantum dots with ferromagnetic leads, Phys. Rev. B 77, 195320 (2008).

[13] A. Aharony, Y. Tokura, G. Z. Cohen, O. Entin-Wohlman, and S. Katsumoto, Filtering and analyzing mobile qubit information via Rashba-Dresselhaus-Aharonov-Bohm interferometers, Phys. Rev. B 84, 035323 (2011) and references therein.

[14] H. Saarikoski, A. A. Reynoso, J. P. Baltanás, D. Frustaglia, and J. Nitta, Spin interferometry in anisotropic spin-orbit fields, Phys. Rev. B 97, 125423 (2018); F. Nagasawa, A. A. Reynoso, J. P. Baltanás, D. Frustaglia, H. Saarikoski, and J. Nitta, Gate-controlled anisotropy in Aharonov-Casher spin interference: Signatures of Dresselhaus spin-orbit inversion and spin phases, Phys. Rev. B 98, 245301 (2018).

[15] P. M. Shmakov, A. P. Dmitriev, and V. Yu. Kachorovskii, High-temperature Aharonov-Bohm-Casher interferometer, Phys. Rev. B 85, 075422 (2012); Aharonov-Bohm conductance of a disordered single-channel quantum ring, Phys. Rev. B 87, 235417 (2013).

[16] F. Nagasawa, J. Takagi, Y. Kunihashi, M. Kohda, and J. Nitta, Experimental Demonstration of Spin Geometric Phase: Radius Dependence of Time-Reversal Aharonov-Casher Oscillations, Phys. Rev. Lett. 108, 086801 (2012).

[17] B. L. Altshuler and L. I. Glazman, Pumping electrons, Science 283, 1864 (1999).

[18] J. E. Avron, A. Elgart, G. M. Graf, and L. Sadun, Geometry, statistics, and asymptotics of quantum pumps, Phys. Rev. B 62, R10618 (2000).

[19] D. J. Thouless, Quantization of particle transport, Phys. Rev. B 27, 6083 (1983).

[20] P. Sharma and P. W. Brouwer, Mesoscopic Effects in Adiabatic Spin Pumping, Phys. Rev. Lett. 91, 166801 (2003).

[21] M. Governale, F. Taddei, and R. Fazio, Pumping spin with electrical fields, Phys. Rev. B 68, 155324 (2003).

[22] Y. Avishai, D. Cohen, and N. Nagaosa, Purely Electric Spin Pumping in One Dimension, Phys. Rev. Lett. 104, 196601 (2010).

[23] V. Brosco, M. Jerger, P. San-José, G. Zarand. A. Shnirman, and G. Schön, Prediction of resonant all-electric spin pumping with spin-orbit coupling, Phys. Rev. B 82, 041309(R) (2010).

[24] M. Jonson, R. I. Shekhter, O. Entin-Wohlman, A. Aharony, H. C. Park, and D. Radić, DC spin generation by junctions with $A C$ driven spin-orbit interaction, Phys. Rev. B 100, 115406 (2019).

[25] Y. Aharonov and A. Casher, Topological quantum Effects for Neutral Particles, Phys. Rev. Lett. 53, 319 (1984).

[26] Y. Meir, Y. Gefen, and O. Entin-Wohlman, Universal effects of spin-orbit scattering in mesoscopic systems, Phys. Rev. Lett. 63, 798 (1989); Y. Oreg and O. EntinWohlman, Transmissions through low-dimensional mesoscopic systems subject to spin-orbit scattering, Phys. Rev. B 46, 2393 (1992).

[27] A. Aharony, O. Entin-Wohlman, M. Jonson, and R. I. Shekhter, Electric and magnetic gating of Rashba-active weak links, Phys. Rev. B 97, 220404(R) (2018).

[28] O. Entin-Wohlman and A. Aharony, Spin geometricphases in hopping magnetoconductance, Phys. Rev. Research (in press); arXiv:1908.05869.

[29] D. C. Langreth, Linear and nonlinear response theory with applications, in Linear and Nonlinear Electron Transport in Solids, eds. J. T. Devreese and E. van Boren (Plenum, New York, 1976).

[30] A-P. Jauho, Nonequilibrium Green function modelling of 
transport in mesoscopic systems, in Progress in Nonequilibrium Green's Functions II, eds. M. Bonitz and D. Semkat (World Scientific, Singapore, 2003).

[31] A-P. Jauho, N. S. Wingreen, and Y. Meir, Timedependent transport in interacting and noninteracting resonant-tunneling systems, Phys. Rev. B 50, 5528 (1994).

[32] M. M. Odashima and C. H. Lewenkopf, Time-dependent resonant tunneling transport: Keldysh and KadanoffBaym nonequilibrium Green's functions in an analytically soluble problem, Phys. Rev. B 95, 104301 (2017).

[33] See Supplemental Material at [URL will be inserted by publisher] for details of the calculation.

[34] M. Abramowitz and I. A. Stegun, Handbook of mathematical functions, National Bureau of Standards, Applied Mathematics Series - 55 (1964), p. 361.
[35] Trivially, all channels are equally open when $\Omega=0$, in which case $f(\omega+n \Omega)$ is independent of $n$ and $I_{D C}$, given by Eq. (21), vanishes (since $\sum_{n} J_{n}^{2}(x)=1$ [34]).

[36] Z. Scherübl, G. Fülöp, M. H. Madsen, J. Nygard, and S. Csonka, Electrical tuning of Rashba spin-orbit interaction in multigated InAs nanowires, Phys. Rev. B 94, 035444 (2016).

[37] K. C. Nowack, F. H. L. Koppens, Y. V. Nazarov, and L. M. K. Vandersypen, Coherent control of a single electron spin with electric fields, Science (New York, N.Y.) 318, 1430 (2007).

[38] J.-W. Luo, S.-S. Li, and A. Zunger, Emergence of an upper bound to the electric field controlled Rashba spin splitting in InAs nanowires, arXiv:2003.02984v1. 